\title{
Sistema de conhecimento para diagnóstico em acupuntura: uma modelagem usando o CommonKADS
}

\author{
Knowledge system for acupuncture diagnosis: \\ a modeling using CommonKADS
}

\author{
Luiz Fernando Lopes ${ }^{1}$ \\ Maurício Capobianco Lopes ${ }^{1,2}$ \\ Francisco Antonio Pereira Fialho ${ }^{1}$ \\ Alexandre Leopoldo Gonçalves ${ }^{1}$
}

\begin{abstract}
Resumo: A tomada de decisão em acupuntura é um processo complexo, uma vez que existe uma grande variedade de elementos e circunstâncias a serem considerados em um tratamento. Uma tarefa intensiva em conhecimento fundamental para o sucesso do tratamento é o diagnóstico. Assim, este artigo tem por objetivo apresentar uma proposta de sistema de conhecimento, utilizando a metodologia CommonKADS, com o objetivo de auxiliar na tarefa de diagnóstico em acupuntura, minimizando o grau de incerteza do tratamento e melhorando as condições de saúde e bem-estar do paciente. O modelo proposto é validado pela construção de um protótipo para diagnóstico de acne utilizando redes bayesianas.
\end{abstract}

Palavras-chave: Engenharia do conhecimento. Metodologia CommonKADS. Acupuntura. Odontologia. Diagnóstico. Sistemas de conhecimento.

Abstract: Decision making in acupuncture is a complex process, once there is a great variety of elements and circumstances to be considered during the treatment. The diagnosis, a knowledge-intensive task, is fundamental for the success of the treatment. Therefore, this study proposes a knowledge system based on the CommonKADS methodology aiming to support diagnosis tasks and to minimize the level of uncertainty in order to improve patient health conditions and welfare. The proposed model is validated through acne diagnosis prototype using Bayesian networks.

Keywords: Knowledge engineering. CommonKADS methodology. Acupuncture. Ontology. Diagnosis. Knowledge systems.

\section{Introdução}

A Medicina Tradicional Chinesa oferece diversas práticas de saúde voltadas ao bem-estar do organismo, tanto no que diz respeito ao físico quanto ao mental. Uma das práticas mais conhecidas e utilizadas é a acupuntura que tem por finalidade a restauração do funcionamento equilibrado do organismo e a prevenção de patologias e afecções. Trata-se de um método que estimula determinados pontos do corpo com agulhas, com o intuito de restaurar ou manter a saúde (YAMAMURA, 2001).

A acupuntura proporciona uma visão sistêmica complexa sobre o organismo humano, composta de uma grande quantidade de agentes que se interrelacionam e que sofrem influência de elementos internos e externos. Este contexto sistêmico exige um conjunto de conhecimentos que muitas vezes dificulta a realização de um diagnóstico acurado de forma a oferecer um tratamento mais preciso e rápido aos pacientes.

Neste contexto, a Engenharia e a Gestão do Conhecimento dispõem de métodos e técnicas utilizadas na explicitação, uso e disseminação do conhecimento, que podem prover ao acupunturista um conjunto de recursos focados no apoio ao processo de tratamento de seus pacientes. Lukman, He e Hui (2007) apresentam um estudo detalhado sobre métodos computacionais aplicados à Medicina Tradicional Chinesa, destacando bases de dados, ferramentas de mineração e sistemas de diagnóstico. Estes recursos podem apoiar tanto o diagnóstico quanto o acompanhamento do tratamento, visando restabelecer ou manter o equilíbrio sistêmico do organismo.

\footnotetext{
${ }^{1}$ Programa de Pós-Graduação em Engenharia e Gestão do Conhecimento, Universidade Federal de Santa Catarina, Campus Universitário, CP 476, CEP 88040-900, Florianópolis, SC - Brasil, E-mails: 1flopes@utfpr.edu.br; fapfialho@gmail.com; a.1.goncalves@stela.org.br

${ }^{2}$ Departamento de Sistemas e Computação, Universidade Regional de Blumenau, Rua Antonio da Veiga, 140, CEP 89012-900, Blumenau, SC - Brasil, e-mail: mclopes@ furb.br
} 
A necessidade de sistemas que apoiem a atuação do acupunturista é corroborada por Camilloti, Istarel e Chi (2006) que chamam a atenção para a necessidade do profissional dispor de ferramentas baseadas em recursos da tecnologia da informação e comunicação. Estas ferramentas têm o propósito de facilitar o processo decisório no contexto do tratamento de um paciente, uma vez que a acupuntura está fortemente baseada na subjetividade, inexistindo indicadores precisos que permitam avaliar sua eficácia (CAMILLOTI; ISRAEL; CHI, 2006).

Assim, a construção de um sistema de conhecimento neste contexto, deve ser realizada por meio de uma abordagem estruturada, com uma visão organizacional e sistêmica. Um dos recursos disponibilizados pela engenharia do conhecimento para esta finalidade é a metodologia CommonKADS (SCHREIBER et al., 2002).

Sendo assim, o objetivo deste artigo é apresentar a modelagem de um sistema de conhecimento para apoio ao diagnóstico de pacientes de acupuntura, utilizando a metodologia CommonKADS. Este sistema visa oferecer ao acupunturista ferramentas adequadas para realizar o diagnóstico com maior eficácia e precisão, acelerando este processo, e consequentemente proporcionando um tratamento mais adequado, e oferecendo uma melhor qualidade de vida e saúde ao paciente. Para a validação do modelo proposto, é apresentado um protótipo para diagnóstico de acne utilizando redes bayesianas.

\section{Acupuntura}

A acupuntura desperta cada vez mais interesse pelas chamadas práticas complementares ou alternativas nos serviços de saúde no Brasil e encontra-se regulamentada, em termos da sua utilização, pela portaria $n^{\circ}$ 971/2006 do Ministério da Saúde.

A palavra acupuntura é originária do latim, no qual acus significa agulha e punctura significa, literalmente, picar. Consiste, portanto, em inserir agulhas em pontos estratégicos na pele para obter uma resposta terapêutica eficiente.

Utilizando uma linguagem que associa as leis da natureza e a perfeita harmonia entre as partes do organismo, a acupuntura busca a integridade entre o homem e o meio em que vive. O fundamento está nas teorias do Yin-Yang (divisão do mundo em duas forças, interpretando todos os fenômenos complementares), dos cinco movimentos (água, madeira, fogo, terra e metal) e dos sistemas internos. As forças Yin-Yang são reguladas por um princípio chamado Tao (taoísmo - caminho) em que, por meio de uma interação dinâmica entre elas, procura-se um equilíbrio, ou seja, a saúde (YAMAMURA, 2001).

De acordo com o taoísmo, quando uma das forças se destaca sobre a outra, ocorre um desequilíbrio e surgem condições para o adoecimento (ERNST;
WHITE, 2001). Diversos fatores podem ocasionar o desequilíbrio, como por exemplo: condições climáticas extremas, emoções excessivas (alegria, tristeza ou raiva), alimentação não balanceada, excesso de trabalho, sedentarismo, atividade física extenuante, excesso ou falta de sexo, etc. (CHONGHUO, 1993).

Para que este desequilíbrio não ocorra, a acupuntura utiliza um conjunto de procedimentos terapêuticos que visam introduzir estímulos, geralmente pela inserção de agulhas filiformes metálicas, em certos lugares anatomicamente definidos, a fim de obter do organismo a recuperação da saúde ou a prevenção de doença (NASIR, 2002).

A acupuntura é um tratamento de baixo custo e de grandes resultados benéficos, promovendo homeostasia, causando uma melhora na circulação do sangue no cérebro e controlando a dor no sistema nervoso central (SIEDENTOPF, 2005). Como desvantagens ou problemas do tratamento com acupuntura, destacam-se o medo da perfuração por agulhas e da contaminação e o preconceito ainda existente da acupuntura como ciência.

Entretanto, com o crescente aumento do uso deste tratamento pela população, cresce também o interesse da comunidade científica no sentido de comprovar suas propriedades terapêuticas, bem como a validação dos processos metodológicos de diagnóstico e tratamento.

O diagnóstico é uma das tarefas mais complexas na Medicina Tradicional Chinesa (MTC). Segundo Lemos (2006, p. 32), o processo de diagnóstico "baseia-se no princípio fundamental de que sintomas e sinais refletem as condições dos órgãos internos e dos canais", podendo não estar necessariamente relacionados com o processo real de uma determinada doença. Portanto, a investigação para o processo de diagnóstico deve estender-se para além de sintomas e sinais relacionados com a queixa (MACIOCIA, 2005).

Para Lemos (2006), no processo de diagnóstico, o interrogatório do paciente ou anamnese é uma etapa fundamental, pois busca sintomas subjetivos explanados pelo doente. Por ser uma tarefa de uso intensivo do conhecimento, a Engenharia e a Gestão do Conhecimento têm muito a contribuir para aprimorar ainda mais este processo em acupuntura.

\section{Engenharia do conhecimento e a metodologia CommonKADS}

A Engenharia do Conhecimento é uma área que tem por objetivo investigar e propor modelos de representação de conhecimento. Inicialmente tratada como uma subárea da Inteligência Artificial na construção de sistemas de conhecimento para a solução de problemas específicos, a Engenharia do Conhecimento transcendeu esta visão ao considerar todo o contexto sistêmico organizacional das atividades intensivas em conhecimento. 
Assim, a Engenharia do Conhecimento deve prover um conjunto de ferramentas que forneçam suporte à gestão do conhecimento a partir da formalização e explicitação das atividades intensivas em conhecimento nas organizações (SCHREIBER et al., 2002). Para isto, Studer, Benjamins e Fensel (1998) descrevem como fundamental a aplicação de metodologias, teorias, métodos, técnicas e ferramentas voltadas à modelagem do conhecimento e sua consequente representação em sistemas de conhecimento.

Neste contexto, destaca-se a metodologia CommonKADS (SCHREIBER et al., 2002), a qual oferece um conjunto de etapas para o desenvolvimento de sistemas baseados em conhecimento. A metodologia é baseada em quatro princípios que fundamentam a nova Engenharia do Conhecimento (SCHREIBER et al., 2002):

a) A Engenharia do Conhecimento aborda diferentes aspectos dos modelos humanos de conhecimento e não apenas a extração do conhecimento de um especialista;

b) A modelagem do conhecimento deve estar focada inicialmente na estrutura conceitual e não nos detalhes de programação dos sistemas de conhecimento;

c) O conhecimento tem uma estrutura interna estável, podendo ser categorizado em tipos ou papéis;

d) Um projeto de sistema de conhecimento deve ser construído de forma espiral, a partir de uma metodologia que permita este tipo de abordagem.

Além disso, três questões são fundamentais no processo de modelagem de um sistema de conhecimento, utilizando-se da metodologia CommonKADS (SCHREIBER et al., 2002): Por quê? O quê? Como? Para responder a estas questões, a metodologia é organizada em três níveis de modelos (SCHREIBER et al., 2002):

a) Nível de contexto: tem por objetivo identificar os elementos fundamentais do ambiente e do contexto do sistema de conhecimento; b) Nível de conceito: tem por objetivo explicitar e formalizar o conhecimento, bem como destacar como ocorrem as interações entre os agentes envolvidos em sua utilização;

c) Nível de projeto: tem por objetivo apresentar todos os detalhamentos necessários para a construção de um sistema de conhecimento.

A Figura 1 apresenta os modelos de cada um dos níveis do CommonKADS.

O Quadro 1 apresenta uma descrição mais detalhada dos níveis do CommonKADS, para a construção de sistemas de conhecimento, bem como sobre os modelos a serem construídos em cada um dos níveis.

Dos três níveis apresentados, destaca-se o nível de contexto. Este é a principal contribuição do CommonKADS para a Engenharia do Conhecimento, uma vez que não está focado apenas na construção de um sistema, mas, sobretudo, no entendimento do contexto e das relações sistêmicas e organizacionais

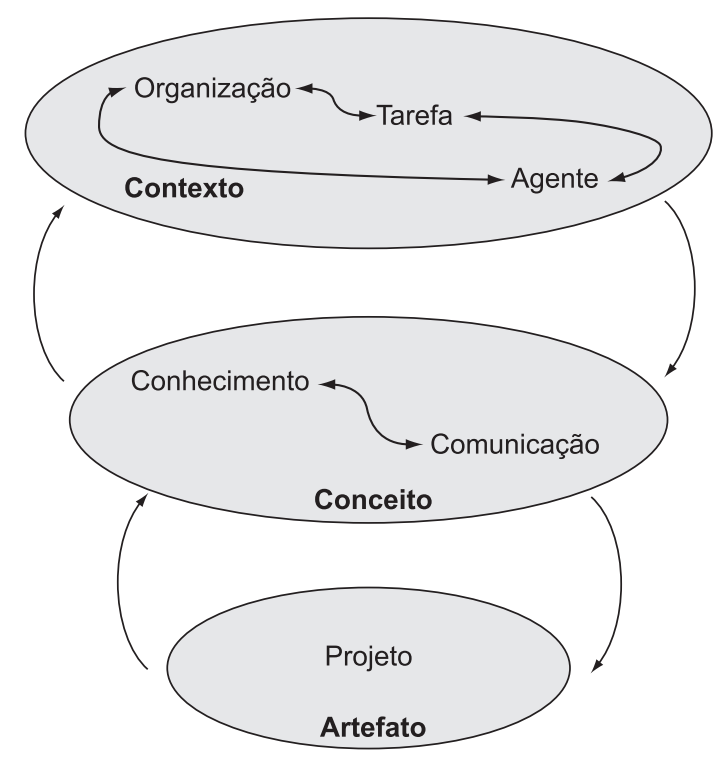

Figura 1. Metodologia CommonKADS. Fonte: adaptado de Schreiber et al. (2002).

Quadro 1. Descrição dos modelos do CommonKADS (SCHREIBER et al., 2002).

\begin{tabular}{|c|c|c|}
\hline Nível & Modelo & Objetivo \\
\hline \multirow[t]{3}{*}{ Contexto } & Organização & Identificar e analisar o contexto organizacional no qual o sistema estará inserido. \\
\hline & Tarefa & $\begin{array}{l}\text { Detalhar todas as tarefas envolvidas no sistema, com foco nas atividades intensivas em } \\
\text { conhecimento. }\end{array}$ \\
\hline & Agente & $\begin{array}{l}\text { Identificar os agentes humanos e não humanos e suas responsabilidades no sistema de } \\
\text { conhecimento. }\end{array}$ \\
\hline \multirow[t]{2}{*}{ Conceito } & Conhecimento & $\begin{array}{l}\text { Apresentar detalhadamente o modelo conceitual de conhecimento do sistema, } \\
\text { destacando as tarefas, as inferências e o domínio para sua posterior implementação. }\end{array}$ \\
\hline & Comunicação & Modelar a comunicação entre os agentes envolvidos no funcionamento do sistema. \\
\hline Artefato & Projeto & $\begin{array}{l}\text { Definir as especificações técnicas necessárias para a implementação e funcionamento } \\
\text { do sistema, com base nas etapas anteriores, }\end{array}$ \\
\hline
\end{tabular}


em que estará inserido. Uma das tarefas fundamentais, e que de certa forma conclui este nível, é o estudo de viabilidade e impacto, tanto no que diz respeito ao negócio quanto às questões técnicas envolvidas.

A seguir será apresentado o desenvolvimento do sistema de conhecimento para acupuntura baseado na metodologia CommonKADS.

\section{Sistema de conhecimento para diagnóstico em acupuntura}

Muitos já são os trabalhos desenvolvidos de sistemas de conhecimento para diagnóstico (BAUMEISTER, 2004; MILNE, 1987; LUO et al., 2006). Segundo Kleer e Williams (1987), entende-se por diagnóstico o processo que visa determinar porque um sistema corretamente projetado não funciona conforme o esperado. Assim, para realizar a tarefa de diagnóstico é necessário observar e levantar hipóteses de modo a chegar a um diagnóstico (resultado), descrevendo o estado do processo observado. De forma simplificada, Schreiber et al. (2002) destacam que o objetivo do diagnóstico é encontrar a causa do mau funcionamento de um sistema.

Neste sentido, este trabalho apresenta um sistema de conhecimento para o diagnóstico em acupuntura, seguindo as etapas da metodologia CommonKADS. São apresentados, entretanto, apenas o modelo de contexto (organização, tarefas e agentes) e o modelo de conceito (conhecimento e comunicação). O modelo de projeto não está contemplado neste trabalho.

\subsection{Modelo de organização}

O modelo de organização é composto de um conjunto de planilhas com a finalidade de detalhar o contexto do sistema de conhecimento na organização. Estas planilhas serão apresentadas nos Quadros 2 a 6 . A primeira planilha é denominada de MO1 - Problemas e oportunidades (Quadro 2). Nesta planilha o problema é tratado com base em uma visão organizacional para além do processo de diagnóstico, uma vez que a acupuntura envolve outras etapas.

A planilha seguinte é denominada de MO2 Aspectos variantes (Quadro 3). Nela são descritos elementos organizacionais que devem ser considerados pelo engenheiro do conhecimento. Como o foco do trabalho é no diagnóstico, são considerados apenas os elementos relevantes no contexto desta tarefa.

A terceira planilha é a MO3 - Detalhamento dos processos (Quadro 4) em que são destacadas as

Quadro 2. MO1: Problemas e oportunidades.

\begin{tabular}{|c|c|}
\hline Modelo organizacional & MO1 - Problemas e oportunidades \\
\hline Problemas e oportunidades & $\begin{array}{l}\text { Problemas: } \\
\text { Dificuldade no diagnóstico e acompanhamento para o tratamento baseado na } \\
\text { acupuntura. } \\
\text { Grande quantidade de pontos existentes para aplicação das agulhas relacionados a } \\
\text { diferentes patologias. } \\
\text { Oportunidades: } \\
\text { Maior precisão no diagnóstico diminuindo o grau de incerteza no tratamento. } \\
\text { Definição de indicadores de acompanhamento do tratamento, visando um } \\
\text { monitoramento mais preciso das condições globais de saúde do paciente. } \\
\text { Definição de indicadores globais para a organização na qual o acupunturista atua } \\
\text { de modo a se fazer a gestão do conhecimento sobre todo o processo organizacional } \\
\text { envolvido como o tratamento dos pacientes. } \\
\text { Disponibilização de uma base de conhecimento sobre tratamentos por meio da } \\
\text { acupuntura. } \\
\text { Melhoria da qualidade de vida do paciente. } \\
\text { Auxílio na capacitação de novos profissionais nesta área. }\end{array}$ \\
\hline Contexto organizacional & $\begin{array}{l}\text { A Acupuntura é normalmente realizada em clínicas autorizadas, por profissionais } \\
\text { habilitados com registro no seu órgão de classe dependendo de sua área de formação. } \\
\text { No Brasil, a atividade de acupuntura vem sendo reivindicada pelos diversos órgãos de } \\
\text { classe que tratam da saúde, no sentido de ser uma especialidade específica de sua área. } \\
\text { Assim, cada conselho adota seus critérios e normas específicos para regulamentar a } \\
\text { atuação do profissional. } \\
\text { Muitas clínicas de saúde utilizam a acupuntura como um tratamento alternativo ou } \\
\text { complementar aos tratamentos convencionais, buscando conquistar novos clientes, } \\
\text { interessados nesta técnica. } \\
\text { Um acupunturista pode atender a vários pacientes em uma mesma sessão, o que, por } \\
\text { um lado, amplia suas possibilidades de ganho financeiro, mas, por outro lado, dificulta } \\
\text { o acompanhamento individual dos pacientes. }\end{array}$ \\
\hline Soluções & Estruturar um sistema de conhecimento para o profissional de Acupuntura. \\
\hline
\end{tabular}


Quadro 3. MO2: Aspectos variantes.

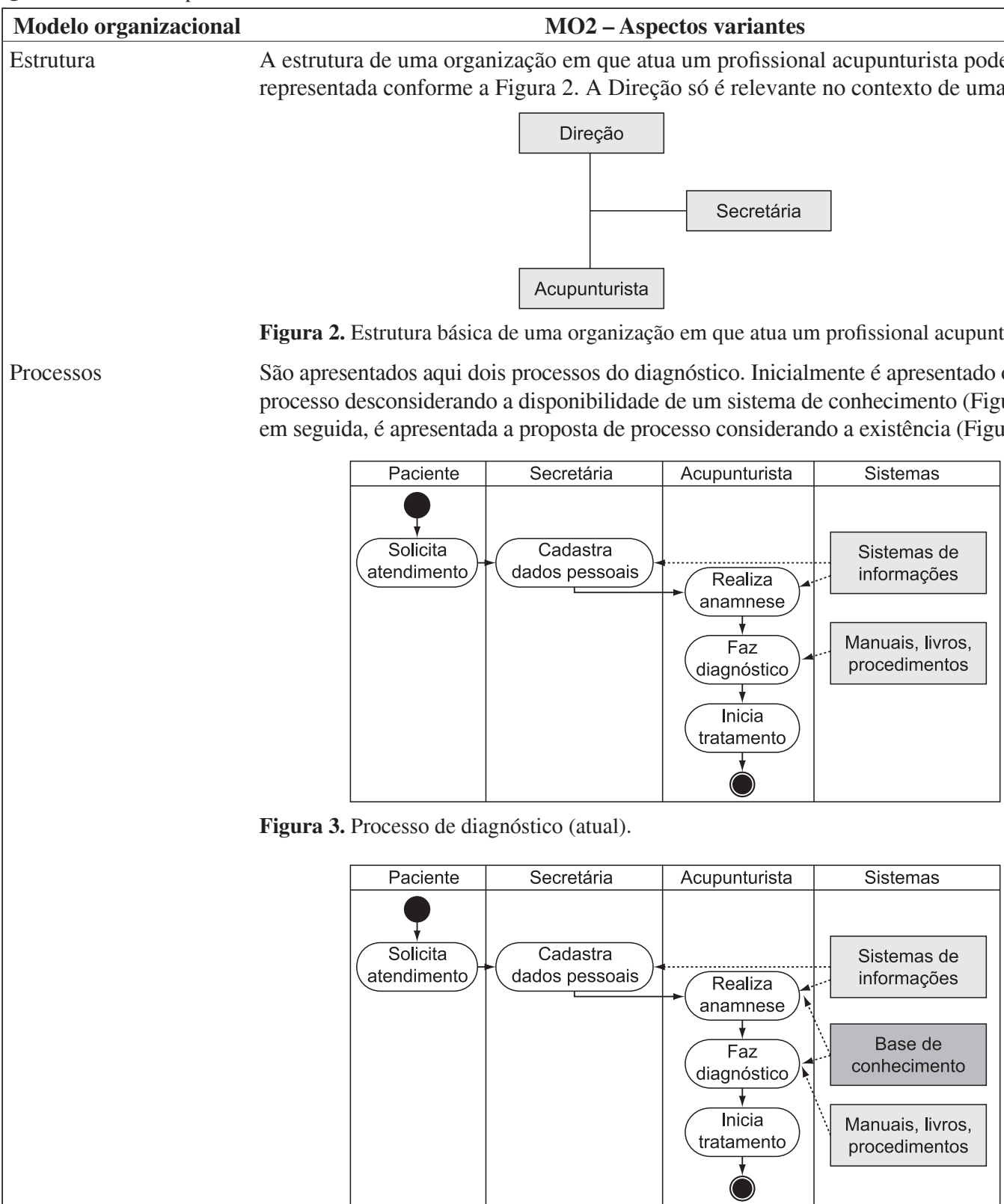

Pessoas

Recursos

Conhecimento

Cultura e poder
Figura 4. Processo de diagnóstico (proposto).

Os principais agentes envolvidos nos processos relatados com suas respectivas atribuições estão descritos a seguir:

Paciente: gera a demanda (problema/necessidade, para o acupunturista).

Secretária: obtém as informações preliminares do paciente.

Acupunturista: realiza o diagnóstico.

Os recursos necessários na atividade do acupunturista são manuais e livros. Na proposta apresentada, pretende-se incluir computador, sistema de informações e sistema de conhecimento.

Os conhecimentos fundamentais para as atividades de acupuntura estão relacionados com as patologias e seus respectivos tratamentos. São conhecimentos complexos que envolvem um contexto sistêmico relacionado ao organismo humano. Normalmente é adquirido por meio de estudos profundos e da prática de sua utilização.

$\mathrm{O}$ acupunturista deve buscar a confiança e empatia do paciente, na busca de uma relação harmônica, pois muitos pacientes têm receio do tratamento, uma vez que ele age diretamente sobre o corpo com um instrumento associado à dor. 
atividades realizadas no contexto da tarefa analisada. $\mathrm{O}$ foco principal é identificar as atividades intensivas em conhecimento e determinar seu significado para o sistema.

A quarta planilha é a MO4 - Ativos de conhecimento (Quadro 5). Nesta planilha o engenheiro procura identificar quais ativos de conhecimento os agentes têm sobre a tarefa.

A quinta e última do modelo de organização é a MO5 - Checklist (Quadro 6) em que o engenheiro estabelece os elementos que definem a viabilidade do sistema de conhecimento a ser construído.

\subsection{Modelo de tarefa}

O modelo de tarefa é composto por um conjunto de planilhas com a finalidade de detalhar as tarefas intensivas em conhecimento identificadas no modelo de organização. Neste trabalho, o foco é apenas na tarefa de diagnóstico. A primeira planilha deste modelo é a TM1 - Análise de tarefa (Quadro 7) que destaca as principais características da tarefa analisada.

Na planilha TM2 - Itens de conhecimento (Quadro 8), o engenheiro faz um checklist sobre o conhecimento da tarefa e identifica pontos possíveis de gargalos e melhorias.

\subsection{Modelo de agente}

O modelo de agente é composto por uma planilha (Quadro 9) que tem a finalidade de apresentar os agentes envolvidos na tarefa. No problema em questão, o único agente tratado é o acupunturista.

Para consolidar o modelo de contexto, a planilha OTA1 (Quadro 10) apresenta um conjunto de informações para auxiliar o processo decisório sobre a implantação do sistema de conhecimento proposto.

A partir do modelo de contexto apresentado até aqui, serão propostos os modelos de conhecimento e comunicação de modo a viabilizar a implantação do sistema.

\subsection{Modelo de conhecimento}

A construção do modelo de conhecimento envolve três etapas (SCHREIBER et al., 2002) que serão detalhadas a seguir.

\subsubsection{Identificação do conhecimento}

Na identificação do conhecimento, duas atividades são fundamentais: a familiarização com o domínio e a lista de potenciais componentes de modelo para reuso. Para isto é fundamental que a tarefa intensiva em conhecimento e os principais itens de conhecimento

Quadro 4. MO3: Detalhamento dos Processos.

\begin{tabular}{|c|c|c|c|c|c|c|}
\hline \multicolumn{2}{|c|}{ Modelo organizacional } & \multicolumn{5}{|c|}{ MO3 - Detalhamento dos processos } \\
\hline $\begin{array}{l}\text { Número } \\
\text { (id) }\end{array}$ & $\begin{array}{c}\text { Tarefa (Nome } \\
\text { da tarefa) }\end{array}$ & $\begin{array}{c}\text { Realizada por } \\
\text { (Agente) }\end{array}$ & Local? & $\begin{array}{c}\text { Ativo de } \\
\text { conhecimento }\end{array}$ & $\begin{array}{l}\text { É intensiva em } \\
\text { conhecimento? }\end{array}$ & Significado \\
\hline 1 & Atendimento & Secretária & $\begin{array}{l}\text { Sala de } \\
\text { espera }\end{array}$ & & Não & 2 \\
\hline 2 & Anamnese & Acupunturista & $\begin{array}{c}\text { Sala de } \\
\text { atendimento }\end{array}$ & $\begin{array}{l}\text { Conhecimentos sobre } \\
\text { os fatos relacionados } \\
\text { com a doença e com o } \\
\text { paciente. }\end{array}$ & Sim & 3 \\
\hline 3 & Diagnóstico & Acupunturista & $\begin{array}{c}\text { Sala de } \\
\text { atendimento }\end{array}$ & $\begin{array}{l}\text { Conhecimentos sobre } \\
\text { as relações entre os } \\
\text { sintomas do paciente } \\
\text { e as possibilidades de } \\
\text { tratamento. }\end{array}$ & Sim & 5 \\
\hline
\end{tabular}

Quadro 5. MO4: Ativos de conhecimento.

\begin{tabular}{|lcccccc|}
\hline \multicolumn{1}{|c}{ Modelo organizacional } & Possuído por & Usado em & $\begin{array}{c}\text { Forma - Ativos de conhecimento } \\
\text { Ativo }\end{array}$ & $\begin{array}{c}\text { Local } \\
\text { correta? } \\
\text { correto? }\end{array}$ & $\begin{array}{c}\text { Tempo } \\
\text { correto? }\end{array}$ & $\begin{array}{c}\text { Qualidade } \\
\text { adequada? }\end{array}$ \\
\hline $\begin{array}{l}\text { Conhecimentos sobre os fatos } \\
\text { relacionados com a doença e } \\
\text { com o paciente }\end{array}$ & Paciente & $\begin{array}{c}\text { Anamnese } \\
\text { Diagnóstico }\end{array}$ & Sim & Sim & Sim & Sim \\
$\begin{array}{l}\text { Conhecimentos sobre as } \\
\text { relações entre os sintomas do } \\
\text { paciente e as possibilidades } \\
\text { de tratamento }\end{array}$ & Acupunturista & Diagnóstico & Sim & Sim & Sim & $\begin{array}{c}\text { Depende da } \\
\text { experiência do } \\
\text { especialista }\end{array}$ \\
\hline
\end{tabular}


Quadro 6. MO5: Checklist para a tomada de decisão de viabilidade.

\begin{tabular}{|c|c|}
\hline Modelo organizacional & MO5 - Checklist para a tomada de decisão de viabilidade \\
\hline Viabilidade de negócio & $\begin{array}{l}\text { A acupuntura apresenta um mercado em expansão, com o crescente interesse de } \\
\text { profissionais da área de saúde, pesquisadores e comunidade científica, os quais têm } \\
\text { realizado diversos trabalhos científicos sobre a técnica, comprovando sua eficácia. } \\
\text { Além disso, a técnica desperta interesse cada vez maior da população como uma forma } \\
\text { de tratamento natural alternativo aos métodos tradicionais baseados na alopatia. } \\
\text { Assim, a análise do contexto organizacional permite verificar a possibilidade de ganhos } \\
\text { por meio da realização de um diagnóstico mais preciso e ágil, o que permite acelerar o } \\
\text { tratamento e acompanhar mais pacientes de uma forma mais eficiente. } \\
\text { Além disso, com a agilidade no tratamento, o paciente obterá melhor qualidade de vida } \\
\text { com condições de recomendar o tratamento para outras pessoas. }\end{array}$ \\
\hline Viabilidade técnica & $\begin{array}{l}\text { Tecnicamente, a construção de um sistema de conhecimento de apoio ao profissional } \\
\text { acupunturista é viável, a partir da evolução da engenharia do conhecimento e das } \\
\text { tecnologias de informação e comunicação. } \\
\text { Como exemplos podem ser citados os modelos de representação do conhecimento } \\
\text { e as redes bayesianas que possibilitam a modelagem e construção de um sistema de } \\
\text { conhecimento com um nível de complexidade exigido pela área de acupuntura. }\end{array}$ \\
\hline Viabilidade do projeto & $\begin{array}{l}\text { A execução do projeto implica no contato com especialistas para a explicitação e } \\
\text { formalização do conhecimento. Como analisado anteriormente na viabilidade técnica, } \\
\text { os recursos tecnológicos estão disponíveis requerendo recursos orçamentários de } \\
\text { pequena monta para sua execução. }\end{array}$ \\
\hline Ações propostas & $\begin{array}{l}\text { A partir do conhecimento de especialistas em acupuntura, pretende-se desenvolver um } \\
\text { sistema de conhecimento para auxiliar no diagnóstico dos pacientes. O diagnóstico } \\
\text { pode ser auxiliado por meio da criação e manutenção de bases de conhecimento. }\end{array}$ \\
\hline
\end{tabular}

Quadro 7. TM1: Análise de tarefa.

\begin{tabular}{|c|c|}
\hline Modelo de tarefa & TM1 - Análise de tarefa \\
\hline Tarefa & Diagnóstico \\
\hline Organização & $\begin{array}{l}\text { Corresponde à terceira etapa do processo de atendimento do paciente e é executada pelo } \\
\text { acupunturista. }\end{array}$ \\
\hline $\begin{array}{l}\text { Objetivo ou valor } \\
\text { agregado }\end{array}$ & Identificar as condições de saúde e os desequilíbrios do organismo do paciente. \\
\hline $\begin{array}{l}\text { Dependências e } \\
\text { fluxo }\end{array}$ & $\begin{array}{l}\text { Tarefa de entrada: anamnese. } \\
\text { Tarefa de saída: tratamento. }\end{array}$ \\
\hline $\begin{array}{l}\text { Objetos } \\
\text { manuseados }\end{array}$ & $\begin{array}{l}\text { Objetos de Entrada: dados do paciente, resultados da anamnese, livros e estudos sobre } \\
\text { acupuntura. } \\
\text { Objeto de Saída: diagnóstico da(s) patologia(s) e indicações de tratamento. } \\
\text { Objetos internos: experiência do acupunturista. }\end{array}$ \\
\hline $\begin{array}{l}\text { Tempo e } \\
\text { controles }\end{array}$ & $\begin{array}{l}\text { O diagnóstico é executado previamente ao tratamento, mas pode ser retomado se o tratamento } \\
\text { não produzir os resultados esperados. Não há um tempo pré-estabelecido tanto para a duração } \\
\text { quanto para a frequência em que ocorre esta tarefa. } \\
\text { Pré-condições: a anamnese conseguir identificar adequadamente as condições do paciente. } \\
\text { Pós-condições: condições de iniciar o tratamento. }\end{array}$ \\
\hline Agentes & O Acupunturista faz o diagnóstico. \\
\hline $\begin{array}{l}\text { Conhecimento e } \\
\text { competência }\end{array}$ & $\begin{array}{l}\text { O principal ativo de conhecimento para a realização desta tarefa é o conhecimento e a } \\
\text { experiência do acupunturista. Outro fator relevante é a habilidade humana do acupunturista } \\
\text { que deve procurar estabelecer empatia e conquistar a confiança do paciente. }\end{array}$ \\
\hline Recursos & $\begin{array}{l}\text { Os principais recursos quantificáveis utilizados para a realização da tarefa são os manuais e } \\
\text { livros sobre acupuntura. }\end{array}$ \\
\hline $\begin{array}{l}\text { Qualidade e } \\
\text { desempenho }\end{array}$ & $\begin{array}{l}\text { A medida de qualidade e performance desta tarefa depende da tarefa seguinte que é o } \\
\text { tratamento, ou seja, se o tratamento alcançar os resultados esperados dentro de sua prescrição, } \\
\text { a tarefa de diagnóstico foi executada adequadamente. }\end{array}$ \\
\hline
\end{tabular}


Quadro 8. TM2: Itens de conhecimento.

\begin{tabular}{|c|c|c|}
\hline Modelo de tarefa & \multicolumn{2}{|c|}{ TM2 - Itens de conhecimento } \\
\hline $\begin{array}{l}\text { Nome: Realizar diagnóstico } \\
\text { Pertence ao: Acupunturista }\end{array}$ & \multicolumn{2}{|c|}{$\begin{array}{l}\text { Usado em: Tarefa } 3 \text { - Diagnóstico } \\
\text { Domínio: Acupuntura }\end{array}$} \\
\hline Natureza do conhecimento & & Gargalos/Melhorias \\
\hline \multicolumn{3}{|l|}{ Formal, Rigoroso } \\
\hline Empírico, Quantitativo & $\mathrm{X}$ & $\mathrm{X}$ \\
\hline Heurístico, Regras & $\mathrm{X}$ & \\
\hline Altamente especializada & $\mathrm{X}$ & \\
\hline Baseado em experiência & $\mathrm{X}$ & $\mathrm{X}$ \\
\hline \multicolumn{3}{|l|}{ Baseado em atividades } \\
\hline \multicolumn{3}{|l|}{ Incompleto } \\
\hline Incerto, pode estar incorreto & $\mathrm{X}$ & \\
\hline \multicolumn{3}{|l|}{ Mudando rapidamente } \\
\hline \multicolumn{3}{|l|}{ Difícil de verificar } \\
\hline \multicolumn{3}{|l|}{ Tácito, difícil de transmitir } \\
\hline \multicolumn{3}{|l|}{ Formato do conhecimento } \\
\hline Mente & $\mathrm{X}$ & $\mathrm{X}$ \\
\hline Papel & $\mathrm{X}$ & \\
\hline Formato eletrônico & $\mathrm{X}$ & \\
\hline Habilidade na ação & $\mathrm{X}$ & \\
\hline \multicolumn{3}{|l|}{ Disponibilidade do conhecimento } \\
\hline \multicolumn{3}{|l|}{ Limitações de tempo } \\
\hline \multicolumn{3}{|l|}{ Limitações de espaço } \\
\hline \multicolumn{3}{|l|}{ Limitações de acesso } \\
\hline \multicolumn{3}{|l|}{ Limitações de qualidade } \\
\hline Limitações de forma & & \\
\hline
\end{tabular}

Quadro 9. AM1: agente.

\begin{tabular}{|ll|}
\hline \multicolumn{1}{|c|}{ Modelo de Agentes } & \multicolumn{1}{c|}{ AM1 - Agente } \\
\hline Nome & Acupunturista \\
Organização & $\begin{array}{l}\text { O Acupunturista normalmente está subordinado a uma direção em uma organização de } \\
\text { saúde, mas tem autonomia para realizar a tarefa de diagnóstico. Trata-se de um agente } \\
\text { humano. }\end{array}$ \\
Está envolvido em & $\begin{array}{l}\text { 2- Anamnese } \\
\text { 3- Diagnóstico }\end{array}$ \\
Comunica-se com & $\begin{array}{l}\text { Secretária } \\
\text { Pacientes }\end{array}$ \\
Conhecimento & Acupuntura (diagnóstico) \\
Outras competências & $\begin{array}{l}\text { Habilidade humana procurando estabelecer empatia e conquistar a confiança do } \\
\text { paciente. } \\
\text { Responsabilidades: fazer o diagnóstico. } \\
\text { Responsabilidades e }\end{array}$ \\
regras & $\begin{array}{l}\text { Restrições: submete-se às normas e procedimentos definidos tanto pela organização } \\
\text { em que está inserido quanto pelos conselhos de classe competentes. }\end{array}$ \\
\hline
\end{tabular}


Quadro 10. OTA1: Checklist para decisão sobre impactos e melhorias.

\begin{tabular}{|c|c|}
\hline $\begin{array}{c}\text { Modelo de organização, } \\
\text { tarefa e agente }\end{array}$ & OTA1 - Checklist para decisão sobre impactos e melhorias \\
\hline $\begin{array}{l}\text { Impactos e mudanças na } \\
\text { organização }\end{array}$ & $\begin{array}{l}\text { Estrutura: não há impactos e mudanças necessárias na estrutura da } \\
\text { organização. } \\
\text { Processos: diagnóstico será realizado apoiado por um sistema de } \\
\text { conhecimento, proporcionando maior eficácia e qualidade ao processo. } \\
\text { Recursos: será necessária a utilização de um sistema de conhecimento } \\
\text { disponível em um computador. } \\
\text { Pessoas: o acupunturista terá a seu dispor um sistema de conhecimento, não } \\
\text { existindo outros impactos ou mudanças para as pessoas na organização. } \\
\text { Conhecimento: o conhecimento que anteriormente estava apenas no domínio } \\
\text { do especialista, passa a estar formalizado e explicitado por um sistema de } \\
\text { conhecimento e pode ser compartilhado entre especialistas, profissionais e } \\
\text { estudantes da área. } \\
\text { Cultura e poder: o sistema de conhecimento pode impactar positivamente para } \\
\text { a organização na medida em que ele possibilitará gerar novos conhecimentos } \\
\text { sobre os pacientes e sobre os processos de diagnóstico e tratamento. Permitirá } \\
\text { maior compartilhamento de informações, possibilitando que um paciente possa } \\
\text { ser acompanhado por mais de um profissional. }\end{array}$ \\
\hline $\begin{array}{l}\text { Impactos e mudanças } \\
\text { na tarefa e nos agentes }\end{array}$ & $\begin{array}{l}\text { O acupunturista, de posse de um sistema de conhecimento que auxilie no } \\
\text { diagnóstico, poderá tomar decisões mais rapidamente e com maior precisão, } \\
\text { reduzindo o grau de incerteza e o tempo de execução da tarefa. Com isso, com } \\
\text { o mesmo tempo que ele tem disponível para atendimento na situação atual, } \\
\text { ele poderá atender a um maior número de pacientes, a partir da implantação } \\
\text { da solução proposta. O sistema também pode ser utilizado como fonte de } \\
\text { conhecimento e formação para novos profissionais que desejam atuar na área. } \\
\text { Para os pacientes, espera-se que o tratamento torne-se mais eficaz, melhorando } \\
\text { mais rapidamente sua qualidade de vida. }\end{array}$ \\
\hline Atitudes e compromissos & $\begin{array}{l}\text { O acupunturista deve se comprometer a utilizar o sistema de conhecimento, } \\
\text { como fonte primordial para o seu processo decisório, fazendo sugestões e } \\
\text { críticas para revisão e aperfeiçoamento. }\end{array}$ \\
\hline Ações propostas & $\begin{array}{l}\text { Melhoria: implantar um sistema de conhecimento que auxilie o diagnóstico } \\
\text { para o tratamento de um paciente de acupuntura. } \\
\text { Acompanhamento: para a implantação adequada do sistema é fundamental a } \\
\text { validação do sistema com especialistas e treinamento para utilização. } \\
\text { Resultados esperados: espera-se agilizar o processo de tratamento, gerando } \\
\text { com isto ganhos para a organização já destacados anteriormente. Os custos de } \\
\text { implantação são relativamente baixos, em função da disponibilidade técnica e } \\
\text { tecnológica que viabilizam a construção do sistema de conhecimento proposto. }\end{array}$ \\
\hline
\end{tabular}

estejam devidamente identificados, os quais foram apresentados em detalhe nas seções anteriores.

A familiarização com o domínio tem o objetivo de permitir o completo entendimento sobre o problema, por meio da consulta às diferentes fontes de conhecimento disponíveis.

A tarefa intensiva em conhecimento aqui tratada é o diagnóstico de patologias em um paciente de acupuntura, visando determinar a melhor forma de tratamento. Assim, para a realização deste trabalho, as fontes de conhecimento selecionadas foram um especialista em acupuntura e manuais e livros sobre o tema. Com o especialista foram feitas entrevistas não estruturadas, de forma a se identificar os principais conhecimentos tácitos aplicados na tarefa de diagnóstico. Os manuais e livros foram utilizados para se conhecer os principais elementos e técnicas envolvidas na atividade de acupuntura, com ênfase nas informações mais importantes do processo de diagnóstico.

$\mathrm{Na}$ atividade seguinte, realizou-se a identificação dos potenciais modelos de reuso para o diagnóstico nas dimensões de domínio e tarefa. Para o modelo de domínio, optou-se por sua representação baseado em uma simplificação dos diagramas de classe da UML (Unified Modeling Language), pela facilidade de compreensão que proporcionam. Para o modelo de tarefa foram estudados os templates propostos nos capítulos 5 e 6 de Schreiber et al. (2002). A partir 
destas definições, iniciou-se a etapa de especificação do conhecimento.

\subsubsection{Especificação do conhecimento}

O objetivo desta etapa é obter a especificação completa do modelo de conhecimento, envolvendo tarefa, inferência e domínio. A primeira atividade é a seleção do modelo de tarefa a ser utilizado. A partir do estudo dos templates apresentados, foi selecionado o template do capítulo 5 proposto por Schreiber et al. (2002), sendo necessários, entretanto, alguns ajustes detalhados a seguir.

A atividade seguinte é a de construção de um modelo inicial de conhecimento de domínio, que não será descrita aqui, pois, em seguida, apresentaremos o modelo de domínio mais detalhado.

A próxima etapa é a construção completa do modelo de conhecimento, a qual será apresentada a seguir. A abordagem utilizada foi a middle-out, que, parte do modelo de inferência, para, em seguida, propor os modelos de tarefa e domínio. Esta é a abordagem mais indicada quando o modelo de tarefa é bem conhecido (SCHREIBER et al., 2002).

\section{A) Modelo de inferência}

No modelo de tarefa proposto são utilizadas as seguintes inferências:
- Cobrir: esta inferência busca, por meio de um modelo de causas e efeitos, uma possível hipótese para um determinado problema ou reclamação. Assim, a partir de um estado visível, será sugerida uma hipótese para um estado não visível, baseada nos modelos causais. A Figura 5 apresenta a representação desta inferência de forma anotada para o exemplo de acupuntura. No exemplo, a partir da reclamação do paciente que tem um problema de acne (estado visível), esta inferência pode trazer uma hipótese que é a existência de acne em função do calor no estômago e pulmões (estado não visível);

- Especificar: esta inferência especifica um possível estado observável, a partir de uma hipótese. Assim, a partir de um estado não visível, será sugerido um estado esperado para o estado não visível, considerando os modelos de manifestação para este estado não visível. A Figura 6 apresenta a representação desta inferência de forma anotada para o exemplo de acupuntura. No exemplo, a partir da hipótese da inferência cobrir (acne em função do calor no estômago e pulmões), é gerado um resultado esperado $($ pele seca $=$ verdadeiro $)$ que esteja relacionado com a hipótese;

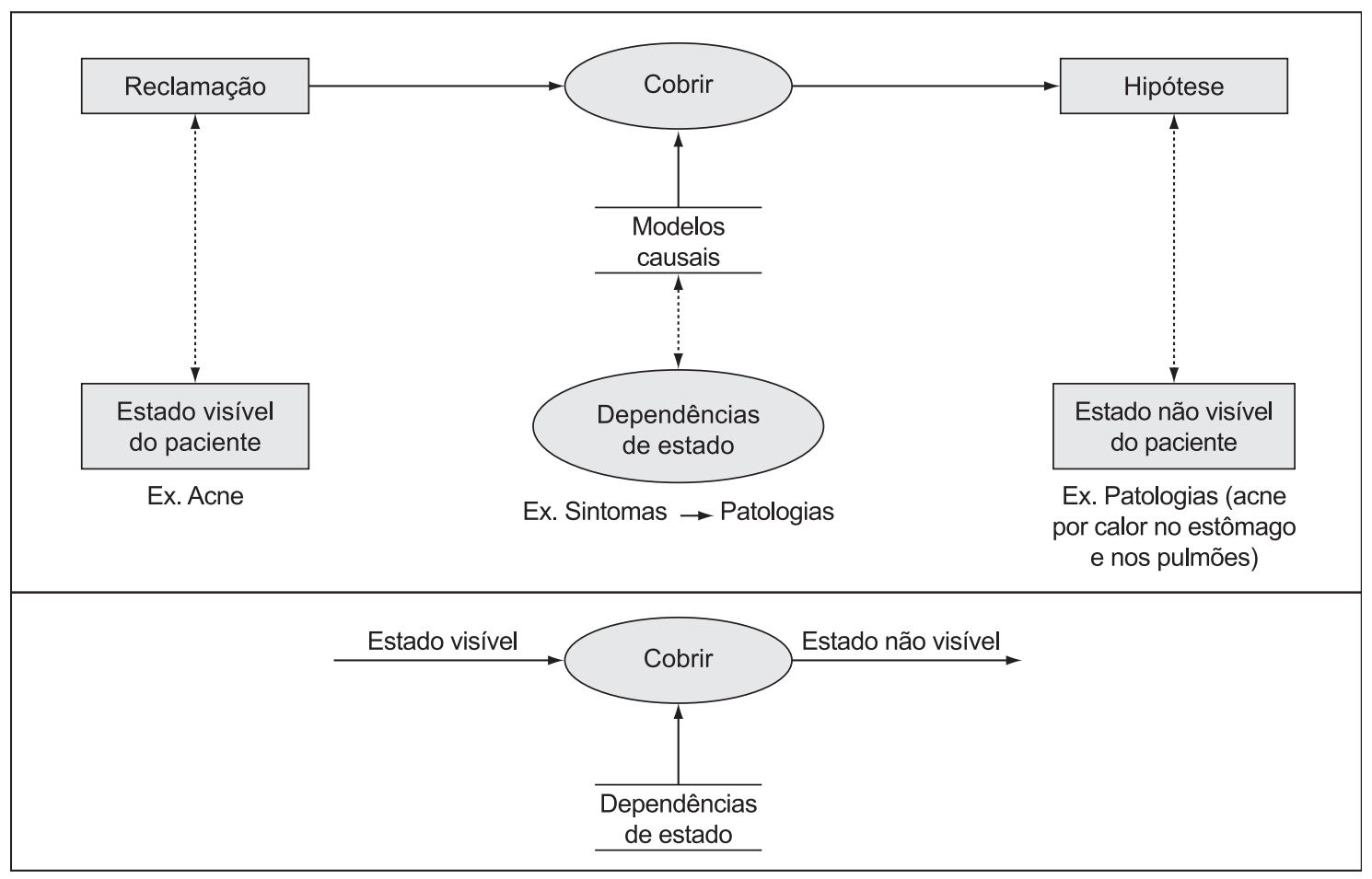

Figura 5. Inferência cobrir. 


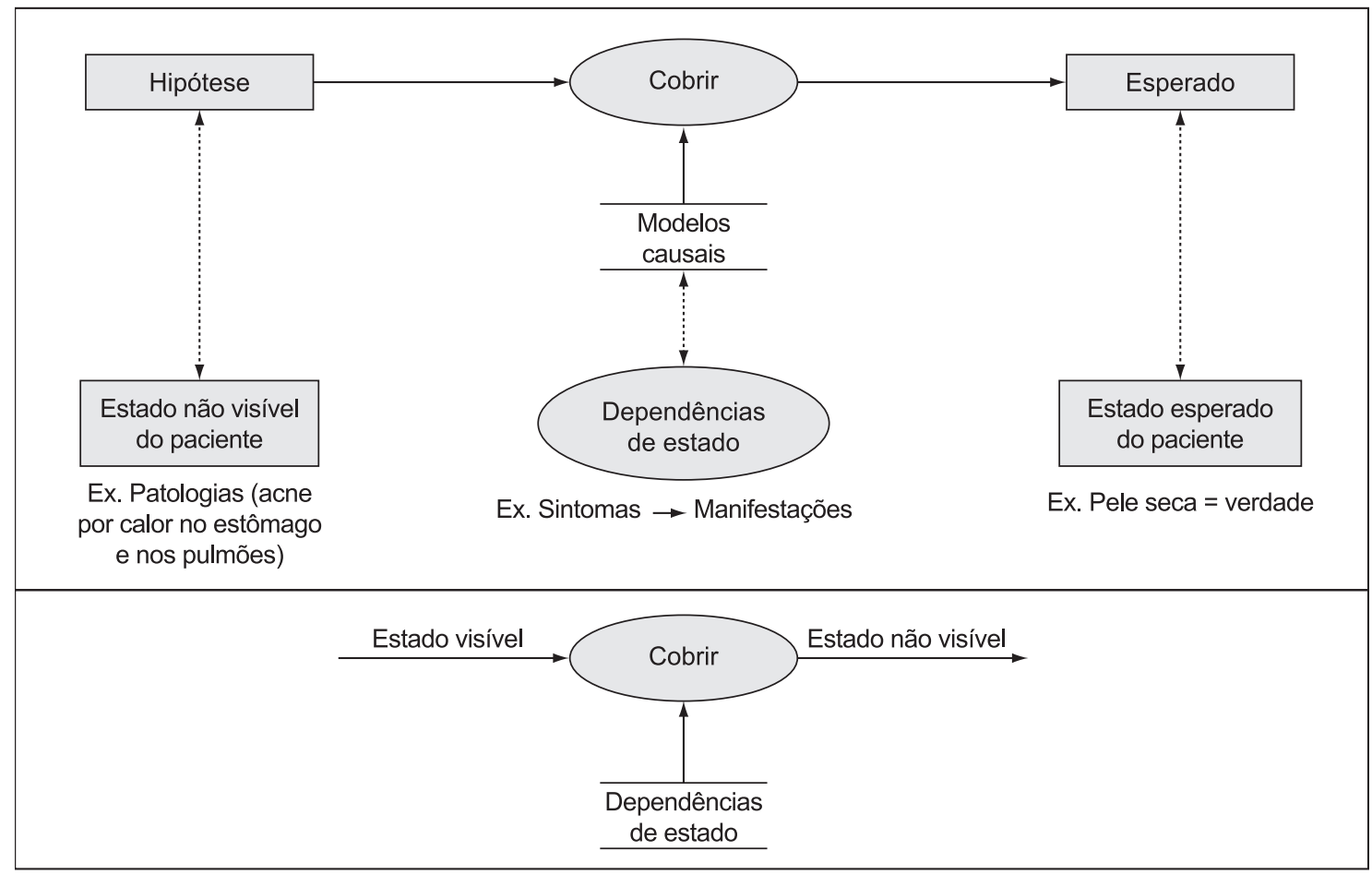

Figura 6. Inferência especificar.

- Obter: trata-se de uma função de transferência, pela qual o acupunturista observa o estado indicado na inferência especificar. A partir do exemplo da Figura 6, ele deverá verificar se o paciente tem pele seca;

- Comparar: esta inferência compara o resultado esperado com o desejado. Assim, a partir do resultado da inferência especificar, o acupunturista deve observar o estado sugerido, por meio da função de transferência obter, de forma a confirmar ou não a hipótese sendo analisada.

\section{B) Modelo de tarefa}

O modelo de tarefa aqui utilizado apresenta modificações em relação ao proposto por Schreiber et al. (2002). A primeira alteração está na troca da inferência predizer pela inferência especificar, tratada anteriormente, uma vez que aquela é pouco adequada ao diagnóstico que se pretende realizar. Outra alteração foi proposta na estrutura de controle com a inserção de um laço de repetição para esta nova inferência. O método de tarefa de diagnóstico a partir de geração e teste está apresentado no Quadro 11 com estas alterações destacadas em negrito.

A Figura 7 apresenta na forma de diagrama de atividades, a estrutura de controle que representa o modelo de tarefa proposto.
A Figura 8 apresenta o modelo de tarefa completo e anotado para o diagnóstico a partir de geração e teste. Através da figura é possível ter uma compreensão global da execução da tarefa, considerando as inferências propostas.

\section{C) Modelo de domínio}

A Figura 9 apresenta o modelo de domínio simplificado, sem considerar os atributos de cada classe existente no sistema.

As classes fundamentais para a tarefa proposta estão descritas a seguir:

a) Paciente: dados e informações pessoais e características do paciente.

b) Sintomas: estados observáveis ou sintomas que podem ser tratados na acupuntura.

c) Anamnese: relaciona paciente e sintomas. São os estados observáveis apresentados por um determinado paciente.

d) Patologias: estados do paciente que podem ser tratados com a acupuntura.

e) Manifestações: relaciona sintomas com patologias. São as manifestações de uma patologia a partir de determinados sintomas.

f) Diagnóstico: relaciona anamnese com manifestações. A partir da anamnese realizada com o paciente e das manifestações existentes para as patologias, constrói-se o diagnóstico. 
Quadro 11. Modelo de tarefa de diagnóstico a partir de geração e teste.

MÉTODO-TAREFA diagnóstico-geração-e-teste;

REALIZA: diagnóstico-paciente;

DECOMPOSIÇÃO:

INFERÊNCIAS: cobrir, especificar, comparar;

FUNÇÃO DE TRANSFERÊNCIA: obter;

PAPÉIS:

INTERMEDIÁRIO:

hipótese: "uma patologia provável";

esperado: "A manifestação da patologia ser verdadeira";

encontrado: "manifestação efetivamente observada";

resultado: "O resultado da comparação da manifestação";

ESTRUTURA DE CONTROLE

ENQUANTO NOVA-SOLUÇÃO cobrir (reclamação $\rightarrow$ hipótese);

FAÇA

resultado == verdadeiro;

ENQUANTO NOVA-SOLUÇÃO especificar (hipótese $\rightarrow$ esperado);

FAÇA

obter (esperado $\rightarrow$ encontrado);

evidência := evidência + encontrado;

comparar (esperado+encontrado $\rightarrow$ resultado);

SE resultado == falso;

ENTÃO "parar a repetição";

FIM SE

FIM ENQUANTO

SE resultado == verdadeiro;

ENTÃO "parar a repetição";

FIM SE

FIM ENQUANTO

$S E$ resultado $==$ verdadeiro

ENTÃO problema-identificado := hipótese;

SENÃO "nenhuma solução encontrada";

FIM SE

FIM MÉTODO-TAREFA diagnóstico-geração-e-teste;

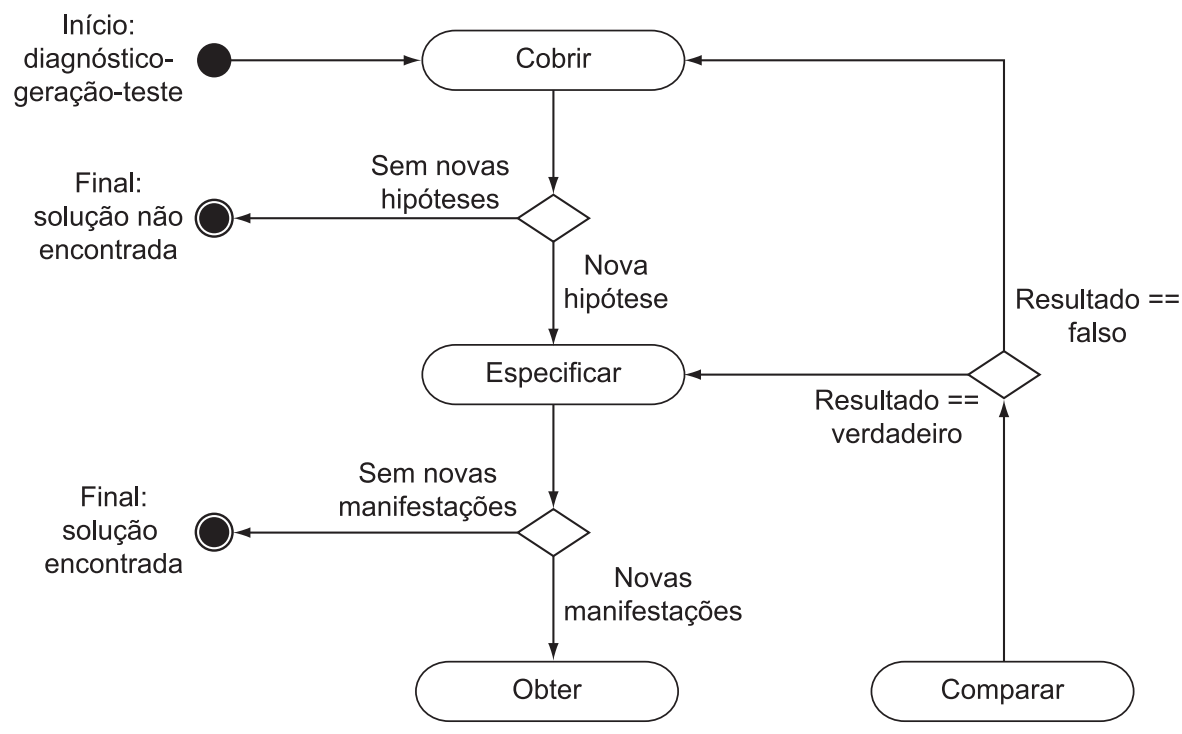

Figura 7. Diagrama de atividades do modelo de tarefa de diagnóstico a partir de geração e teste. 
g) Tratamento: tratamentos realizados com a acupuntura.

f) Tratamento-Patologia: relaciona tratamentos com patologias e com o diagnóstico, indicando para um determinado diagnóstico, qual é o tratamento mais indicado.

As classes de Tratamento e Tratamento-Patologia não têm aplicação no diagnóstico, mas estão representadas apenas para contextualizar o diagnóstico em relação à etapa posterior de acompanhamento do tratamento.

\subsubsection{Refinamento do conhecimento}

A etapa de refinamento do conhecimento tem por objetivo validar os modelos de conhecimento propostos, além de preencher as bases de conhecimento. A validação do modelo de conhecimento consiste em identificar se está consistente, sem ambiguidades e se atende às necessidades para as quais foi concebido.

Para verificar a validade do modelo, foi construído um protótipo simplificado no software Nética (NORSYS NETICA, 2008), utilizando redes bayesianas, que são representações que mapeiam conhecimento a partir de um modelo probabilístico, expressando relações de causas e efeitos, pela associação de probabilidade em um determinado domínio do conhecimento (RUSSEL e NORVIG, 1995).

O exemplo construído tratou do diagnóstico de acne, apresentado nos diagramas da seção anterior. $\mathrm{O}$ protótipo construído contempla apenas uma pequena amostra de todo o conhecimento envolvido no processo de diagnóstico de acne para tratamento por meio da acupuntura. A Figura 10 apresenta o protótipo construído para validação do sistema de conhecimento.

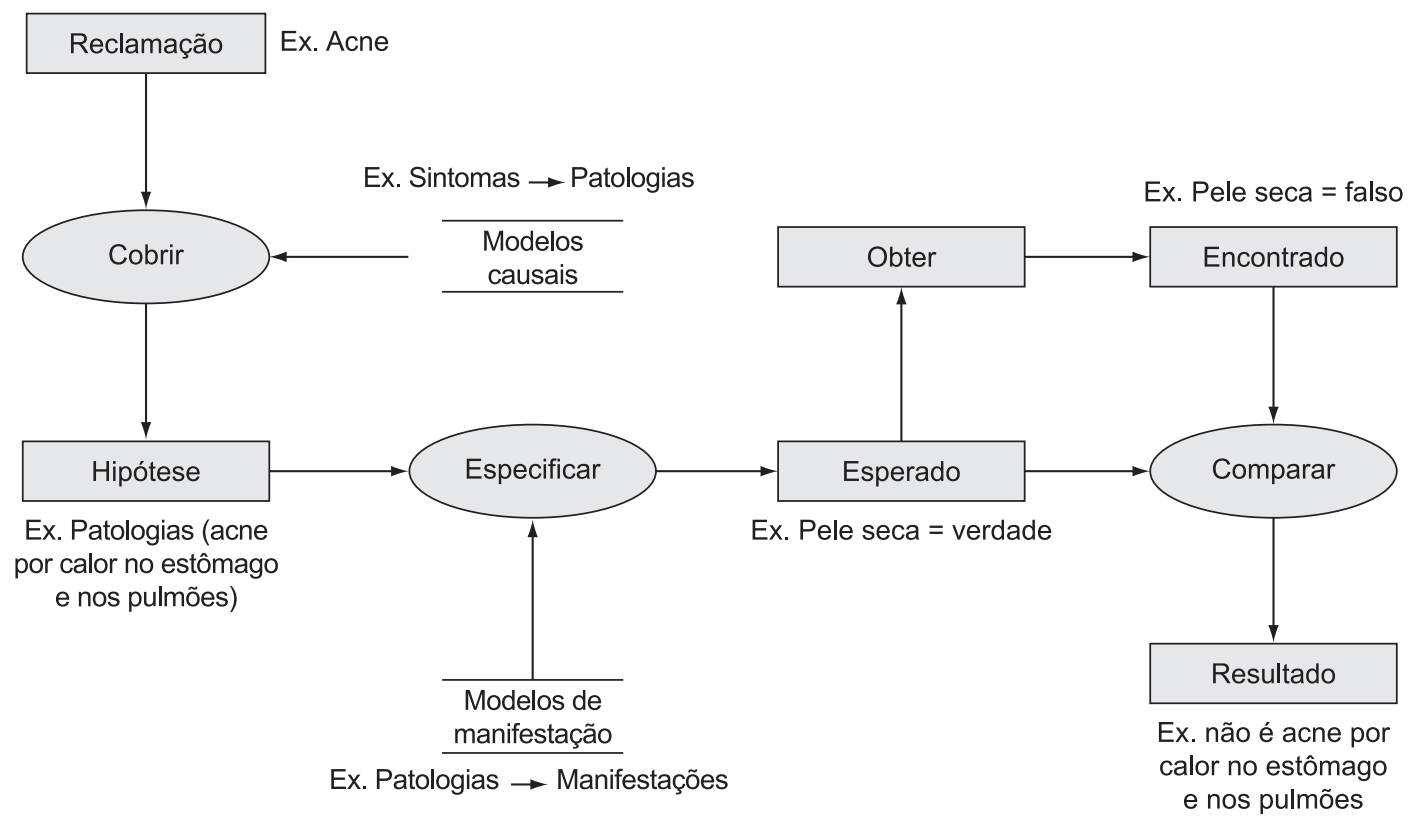

Figura 8. Modelo de tarefa anotado.

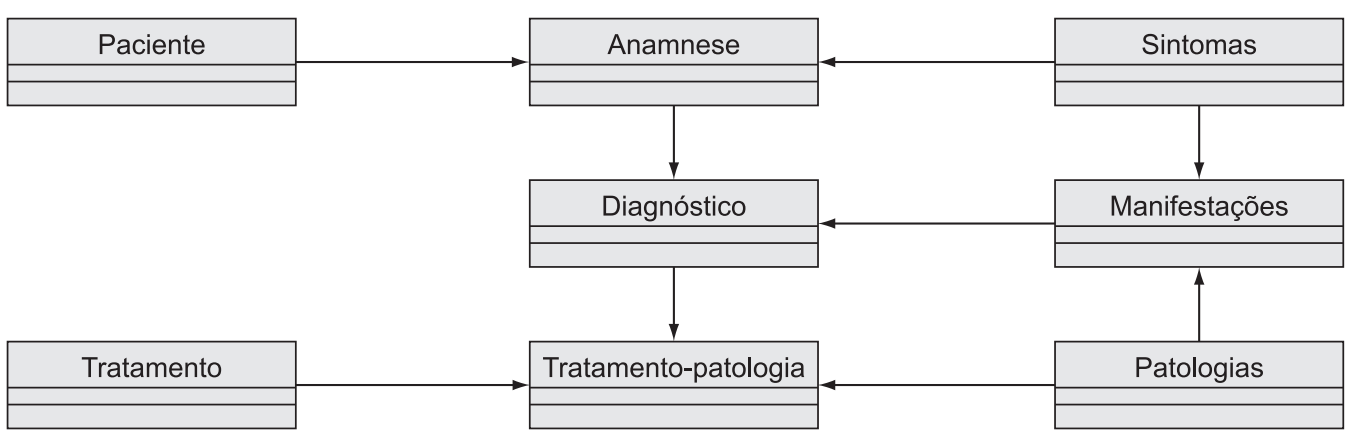

Figura 9. Modelo de domínio simplificado. 


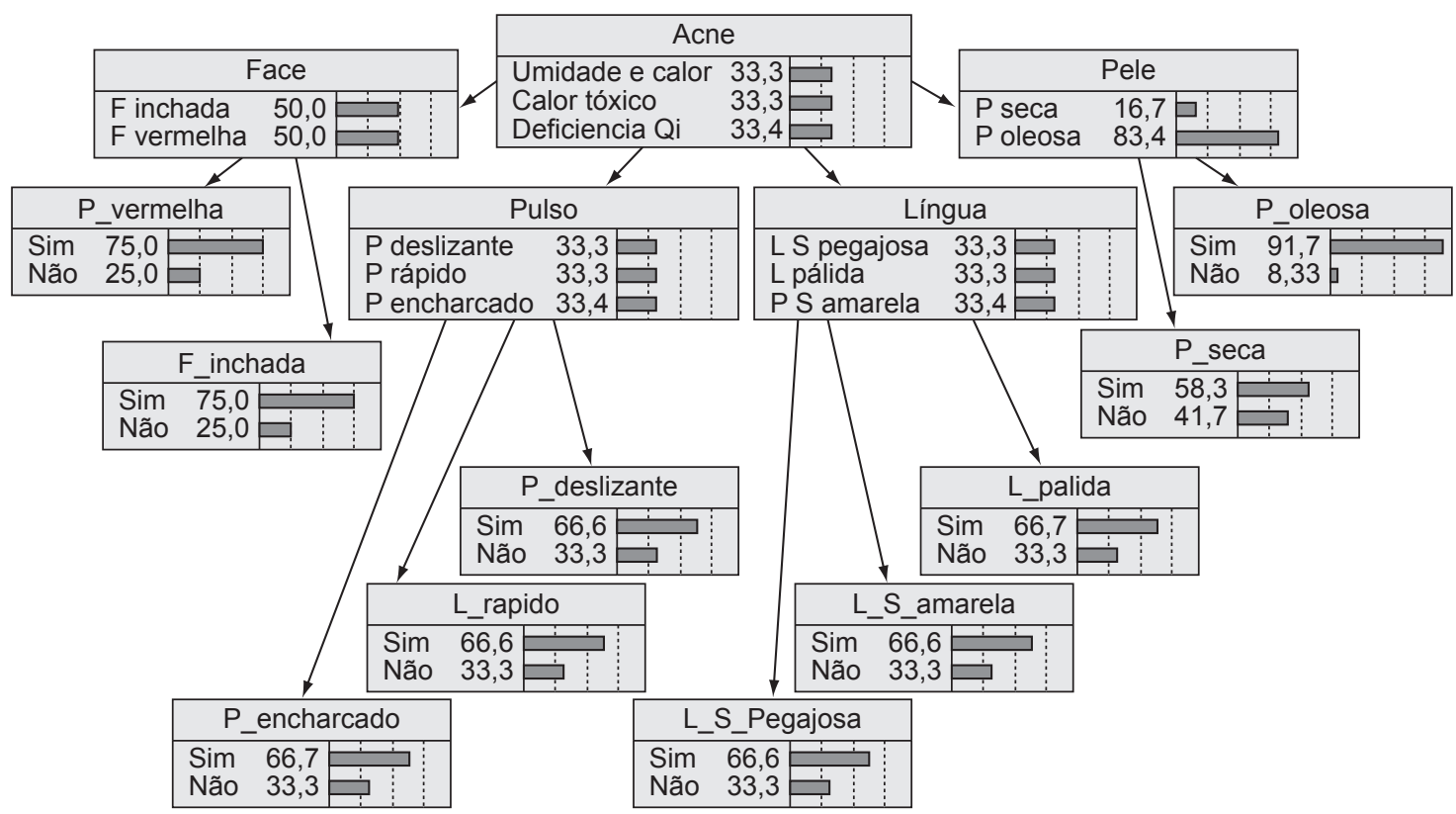

Figura 10. Exemplo de diagnóstico de acne para tratamento por meio de acupuntura usando redes bayesianas.

Quadro 12. Comunicação da tarefa de diagnóstico.

\section{Objetivo:}

Obter o diagnóstico a partir do estado observável apresentado pelo paciente, podendo indicar o tratamento mais adequado.

\section{Pré-condição:}

Ter disponível os dados pessoais do paciente.

Ter realizado a anamnese.

Ter a base de conhecimentos que possibilite diagnosticar a possível patologia do paciente.

\section{Ator:}

Acupunturista

\section{Fluxo principal:}

O Sistema solicita informações sobre o Paciente.

O Acupunturista insere as informações solicitadas no Sistema.

O Sistema executa as inferências a partir das informações repassadas.

O Sistema informa o possível Diagnóstico.

\section{Fluxo alternativo:}

O Sistema não chega a um resultado conclusivo.

Quadro 13. CM1: Descrição da Transação.

\begin{tabular}{|ll|}
\hline \multicolumn{1}{|c|}{ Modelo de comunicação } & \multicolumn{1}{c|}{ CM1 - Descrição de transação } \\
\hline Transação & Obter diagnóstico \\
Objeto de informação & Diagnóstico entre as tarefas de anamnese e tratamento \\
Agentes envolvidos & Acupunturista e Sistema de conhecimento \\
Plano de Comunicação & Ver Quadro 12 \\
Restrições & Ver Quadro 12 \\
Especificação de troca de informação & É uma transação do tipo pergunta e resposta. \\
\hline
\end{tabular}

Os nós folhas representam as informações obtidas por meio da anamnese, ou seja, são as manifestações do organismo que podem causar o desequilíbrio que levam a uma determinada patologia, usados na inferência especificar. No caso da rede bayesiana proposta, as patologias, usadas na inferência cobrir, foram definidas como sendo os atributos do problema reclamado pelo paciente (no caso, Acne). Esta 


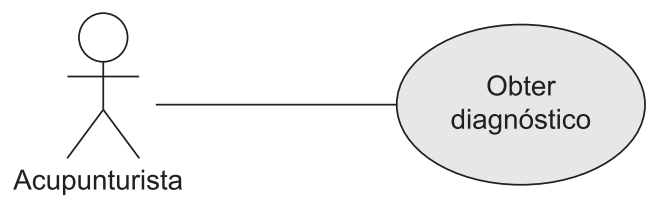

Figura 11. Diagrama de caso de uso para a tarefa de diagnóstico.

abordagem permite, portanto, a partir da anamnese, inferir o tipo de patologia mais provável do paciente, ou seja, com menor grau de incerteza, de forma a indicar o melhor tratamento.

\subsection{Modelo de comunicação}

O modelo de comunicação define como ocorre a troca de informações entre os agentes humanos e não humanos no sistema de conhecimento. Nesta tarefa ele é realizado de forma simples, uma vez que há apenas a interação do acupunturista com o sistema de conhecimento. Desta forma, optou-se por sua representação através de um diagrama de casos de uso da UML, conforme Figura 11 e o Quadro 12.

A planilha CM1 - Descrição de transação (Quadro 13) é utilizada no CommonKADS para apresentar a síntese do modelo de comunicação.

\section{Considerações finais}

O trabalho proposto teve por objetivo apresentar o desenvolvimento de um sistema de conhecimento utilizando a metodologia CommonKADS. A metodologia demonstrou ser adequada para os objetivos propostos na medida em que apresenta uma visão de contexto sistêmico sobre a organização e o problema a ser tratado. Isto a torna menos focada na construção de um artefato, mas sim na construção de um modelo de conhecimento que possa gerar resultados de forma a minimizar as incertezas e riscos e maximizar os ganhos para a organização.

No caso específico deste trabalho, a metodologia foi aplicada a uma clínica especializada em acupuntura, que trata de tarefas intensivas em conhecimento uma vez que o objetivo desta técnica é buscar o equilíbrio do organismo humano, visando dar melhores condições de saúde e bem-estar aos pacientes. O protótipo apresentado, utilizando redes bayesianas, fornece indicativos sobre a viabilidade de construção de um sistema de conhecimento completo para esta finalidade. Esta etapa deverá ser completada futuramente com a execução do modelo de artefato do CommonKADS.

Quando totalmente implementado, pretende-se que o sistema proposto auxilie o acupunturista na tarefa de diagnóstico, visando resultados mais precisos. O sistema também poderá ser estendido para contemplar a tarefa de monitoramento, por meio da criação de indicadores que permitam acompanhar o desenvolvimento e a adequação do tratamento, bem como a precisão do diagnóstico.

\section{Referências}

BAUMEISTER, J. Agile development of diagnostic knowledge systems. IOS Press, 2004.

CAMILLOTI, B. M.; ISRAEL, V. L.; CHI, A. Necessidade de um sistema de apoio à decisão em acupuntura. In: CONGRESSO BRASILEIRO DE INFORMÁTICA EM SAÚDE, 10. 2006, Florianópolis. Anais... SBIS, 2006. Disponível em: <www.sbis.org.br/cbis/arquivos/914. pdf>. Acesso em: 08 set. 2008.

CAMILOTTI, B. M. ; Israel, Vera ; CHI, A. . Necessidade de um Sistema de Apoio à Decisão em Acupuntura. In:. http://www.sbis.org.br/cbis/anaiscbis2006.htm, 2006.

CHONGHUO, T. Tratado de medicina chinesa. São Paulo: Roca, 1993.

ERNST, E.; WHITE, A. Acupuntura: uma avaliação científica. São Paulo: Manole, 2001.

KLEER, J.; WILLIAMS, B. Diagnosing multiple faults. Artificial intelligence, v. 32, n. 1, p. 97-130, 1987.

LEMOS, S. F. Significados de acupuntura por usuários de um serviço de atendimento em saúde. 2006. Dissertação (Mestrado em Ciências da Saúde)-UFG, UnB, UFMS, Goiânia, 2006.

LUKMAN, S.; HE, Y.; HUI, S.-C. Computational methods for traditional Chinese medicine: a survey. Computer Methods and Programs in Biomedicine, v. 88, n. 3, p. 283-294, 2007.

LUO, J. et al. Diagnosis knowledge representation and inference. Instrumentation \& Measurement Magazine, IEEE, v. 9, n. 4, p. 45-52, 2006.

MACIOCIA, G. Diagnóstico na medicina chinesa: um guia geral. São Paulo: Roca, 2005.

MILNE, R. Strategies for diagnosis. Systems, Man and Cybernetics, IEEE Transactions, v. 17, n. 3, 1987.

NASIR, L. S. Acupuncture. Primary Care: Clinics in Office Practice, v. 29, n. 2, p. 393-405, 2002.

NORSYS NETICA: bayesian networks graphical application. [S.1.], 2008. Disponível em: <http://www.norsys.com/ netica.html>. Acesso em: 22 set. 2008.

RUSSEL, S.; NORVIG, P. Artificial intelligence: a modern approach. New Jersey: Prentice-Hall, 1995.

SCHREIBER, G. et al. Knowledge engineering and management: the CommonKADS methodology. Cambridge, Massachusetts: MIT Press, 2002.

SIEDENTOPF, C. M. et al. Laser acupuncture induced specific cerebral cortical and sbcortical activations in humans. Laser in Medical Science, v. 20, p. 68-73, 2005.

STUDER, V. R.; BENJAMINS, R. \& FENSEL, D. Knowledge engineering: principles and methods. Data \& Knowledge Engineering, v. 25, n. 1-2, p. 161-197, 1998.

YAMAMURA, Y. Acupuntura tradicional: a arte de inserir. 2. ed. São Paulo: Roca, 2001 
